$11 \mid 2010$

NOVECENTO... E DINTORNI

Littérature et nouveaux mass médias

\title{
Fratelli coltelli
}

Sur les tumultueuses relations entre cinéma et télévision au pays de Fellini et de Silvio Berlusconi

Oreste Sacchelli

\section{(2) OpenEdition}

\section{Journals}

Édition électronique

URL : http://journals.openedition.org/cei/148

DOI : $10.4000 /$ cei. 148

ISSN : 2260-779X

Éditeur

UGA Éditions/Université Grenoble Alpes

Édition imprimée

Date de publication : 15 juin 2010

Pagination : 251-259

ISBN : 978-2-84310-168-7

ISSN : 1770-9571

\section{Référence électronique}

Oreste Sacchelli, « Fratelli coltelli », Cahiers d'études italiennes [En ligne], 11 | 2010, mis en ligne le 15 décembre 2011, consulté le 27 mars 2021. URL : http://journals.openedition.org/cei/148 ; DOI :

https://doi.org/10.4000/cei.148 
FRATELLI COLTELLI.

\title{
SUR LES TUMULTUEUSES RELATIONS ENTRE \\ CINÉMA ET TÉLÉVISION AU PAYS DE FELLINI \\ ET DE SILVIO BERLUSCONI
}

\author{
Oreste Sacchelli \\ Université de Nancy
}

Partout en Europe, l'apparition de la télévision dans les premières années de l'après-guerre entraîne des chutes de fréquentation des salles de cinéma et un transfert de spectateurs du grand au petit écran, avec des conséquences non négligeables sur les stratégies commerciales des sociétés de télévision et des productions cinématographiques. Il s'agit en fait d'une concurrence pour la conquête du temps libre des individus, la télévision étant soutenue par les industriels du secteur de l'électronique qui voient dans le développement de la consommation du spectacle privé un véritable eldorado. En Italie, ce phénomène est amplifié par la totale mainmise du pouvoir politique sur la Rai dans la cadre du monopole de l'État sur la radio et la télévision, la Démocratie chrétienne entendant en effet utiliser ce nouveau moyen de diffusion de l'information et du divertissement pour promouvoir son modèle de développement économique et social, face à un cinéma considéré frondeur et assez globalement aux mains de l'ennemi politique.

Quelques données chiffrées :

- la diffusion télévisée en Italie commence officiellement le 3 janvier I954. Il n'y a alors que 24000 abonnés;

- c'est en 1955 que le cinéma italien enregistre son record de fréquentation avec 8I9,4 millions de spectateurs, après quoi débute une baisse continue, exactement comme aux États-Unis (4400 millions de spectateurs en 1946) ou en Grande-Bretagne (I 5 I4 millions en 1948) et avant la France (4I2 millions en 1957); 
- en 1959, au début du boom économique, le nombre d'abonnés est de I 572000 et le cinéma a perdu 65 millions de spectateurs.

L'année 1976 représente une rupture encore plus importante. L'arrêt de la cour constitutionnelle qui déclare que le monopole de l'État sur la diffusion par ondes hertziennes est contraire à l'article 2I de la constitution, ouvre ce que l'on a appelé le «far west télévisuel» qui dure pendant toutes les années 1980 et qui verra prospérer Silvio Berlusconi, en l'absence d'une loi de régulation. Lorsque la loi arrive, en 1990 (la fameuse loi Mammì, du nom du ministre des Postes et Télécommunications d'alors), elle ne fait que prendre acte de ce qui existe et régularise de fait le duopolio Rai - Fininvest, la société de Silvio Berlusconi. Le nombre de spectateurs dans les salles de cinéma chute de 513 millions en 1975 à 373 millions en 1977, pour atteindre son minimum historique en 1992 avec 83 millions de spectateurs.

Le monopole de l'État a eu pour effet d'empêcher en Italie l'émergence d'une véritable industrie audiovisuelle qui aurait pu investir dans divers types de programmes et réguler les effets de mutations technologiques qui investissaient le secteur de l'entertainement. Au lieu de cela, les sociétés de production cinématographique ont été limitées au domaine strict du cinéma diffusé en salle et contraintes de chercher des formes nouvelles pour concurrencer la télévision dans le domaine du spectacle populaire. Grosso modo, plus la télévision donnait dans le registre du politiquement correct, plus le cinéma se donnait un aspect politiquement incorrect. C'est ainsi que le genre peplum qui a produit quelque 150 films entre 1956 et 1965 est progressivement remplacé par le western qui se caractérise par une violence et un cynisme que jamais la télévision n'aurait tolérés. C'est par l'excès de violence, de caricature, d'érotisme, que l'industrie cinématographique tente de contrer la télévision. On constate en effet que les mêmes maisons de production, qui changent parfois de nom mais ni de siège ni de personnel, s'investissent successivement dans ces genres pour tenter de conserver le public populaire des salles de seconda visione dans les quartiers, les banlieues et les petites villes.

Un phénomène analogue concerne les salles d'exclusivité (prima visione) des centres des grandes villes qui ont une clientèle plus intellectuelle et soucieuse de qualité cinématographique. Pour cette clientèle, les maisons de production misent sur les auteurs et sur la satire sociale, deux autres déclinaisons de l'excès, que ce soit dans les colosses d'auteur tels que les films de Visconti (particulièrement Il Gattopardo, 1963), ou la caricature féroce des petits bourgeois dans la comédie à l'italienne - où l'on ne manque pas 
de moquer le téléspectateur (Dino Risi et le sketch L'oppio del popolo dans I mostri, 1963).

Toutefois, vis-à-vis de la télévision, tous les cinéastes ne sont pas unanimes. Pasolini et Rossellini représentent les deux attitudes extrêmes qui polarisent, et pour longtemps, le débat. La condamnation pasolinienne de la télévision est connue. Elle entre dans le contexte plus vaste de la condamnation de la société petite-bourgeoise consumériste et conformiste dont la télévision est le principal moyen de propagation. Rossellini, à l'inverse, dès 1957 s’approprie le moyen télévisuel pour résoudre une crise artistique personnelle et se diriger vers la réalisation de films didactiques de vulgarisation. Il réalise ainsi L'India vista da Rossellini pour la RAIRadiotelevisione Italiana (Io episodes, 25I min) et sa version française J'ai fait un beau voyage pour l'ORTF; ce que Fellini commente de la manière suivante :

$H a$, quindi, avuto ragione Rossellini quando ha capito, subito, che il modo più diretto per comunicare e il modo migliore per usare la televisione erano quelli di fare una didascalia illustrata, cioè una sorta di conferenza con diapositive, indicate di volta in volta con un montaggio semplice ${ }^{\mathrm{I}}$.

Cette constatation arrive après une longue analyse des différences fondamentales entre le cinéma et la télévision que Fellini aussi a expérimentée en 1969 (Fellini: a director's notebook pour NBC) et en 1970 (I clowns, une co-production RAI-ORTF-Bavaria Film). Le point de vue de Fellini sur la question est celui de l'auteur cinématographique confronté à un outil trop trivial pour lui permettre de s'exprimer pleinement: la télévision ne permet ni les nuances dans les éclairages ni les profondeurs de champ, fondamentales pour lui. Par ailleurs le dispositif même de la télévision, dit-il, bouleverse totalement le rapport entre l'auteur et le spectateur, privant la communication de tout le rituel de la projection cinématographique qui la sacralise :

Intanto, non è che il pubblico si muova di casa e venga da te. Sei tu che vai da loro: già questo ti mette in una condizione d'inferiorità. E dov'è che vai? Vai forse in questa intimità tanto vagheggiata, per la quale ti sembrava di poter dire che il tuo racconto sarebbe arrivato in una maniera piú diretta? Niente affatto! Infatti, in primo luogo, c'è da vincere l'atteggiamento padronale dello spettatore. Chi ha la televisione è il padrone della televisione. Ciò non accade né in teatro né al cinema: dove lo spettatore non si sente padrone del teatro o del cinema. Anzi: deve uscire di casa, deve fare la fila, deve pagare il biglietto, deve entrare al buio, deve sedersi, dev'essere vestito completamente e non in mutande, oppure in vestaglia, con le pantofole; non può portare i bambini e, se li porta,

I. Federico Fellini, Fare un film, Torino, Einaudi, 1980, p. I40. 
sono obbligati (almeno in teoria) a comportarsi educatamente. C'è insomma un clima di rispetto che dovrebbe predisporre a un certo tipo di ascolto. Alla televisione no. Li sei tu che devi entrare con molta educazione, subito obbligato a interessare o divertire gente che sta a casa sua, quindi sta a tavola, sta mangiando, sta telefonando. Tu autore non puoi ignorare questo fatto e devi perciò essere immediatamente molto divertente o molto interessante, com'erano una volta certi giullari, certi saltimbanchi, in piazza, i quali dovevano attirare l'attenzione di gente che andava per i fatti propri, a spasso, oppure in carrozza, e che alla fine si fermava a guardare da lontano con diffidenza e, comunque, con un atteggiamento di condiscendente tolleranza. Quindi, ripeto, tu devi tener conto che ti rivolgi a un pubblico che va interessato o divertito subito. Perché questo pubblico, questo padrone, poiché ti ha comprato, se non lo diverti immediatamente, ti chiude, o cambia programma, ti spegne e mangia la pastasciutta. Tieni presente inoltre che devi parlare, raccontare le tue storie segrete a gente che, proprio perché si trova in casa propria, ha il pieno diritto di fare tutti $i$ commenti che vuole ad alta voce, e persino di insultarti o, peggio, di ignorarti. Allora, come è possibile rimanere se stessi, essere fedeli al proprio mondo, ai propri "stilemi», in una situazione simile: sapendo, cioè, che devi fare del chiasso per attirare l'attenzione, che sei costretto a dire le cose piu divertenti subito, che non devi perdere tempo, ecc.? Io non credo che sia possibile. Ecco, quindi, che colui che si accinge a parlare per televisione deve tener conto di questo clima, di questa morfologia della comunicazione. Cioè, ripetiamo: assenza del necessario rituale e prevaricazione a rovescio, da parte del pubblico. Il telespettatore, insomma, è il padrone della televisione: infatti, se vuole, può buttarla anche dalla finestra ${ }^{2}$.

Difficile d'être plus clair. On ne s'étonnera donc pas de trouver Federico Fellini à la tête du mouvement de protestation contre les coupures publicitaires dont les films sont l'objet sur les chaînes privées. En effet, le I8 novembre 1988, le jeune député Walter Veltroni présente au parlement au nom du PCI un projet de loi visant à réglementer la télévision commerciale qui agit encore dans le vide juridique le plus total. Le texte prévoit la suppression pure et simple des interruptions publicitaires, reléguant les spots avant, après ou dans la coupure entre les deux parties du film. Le lendemain, le journal l'Unità publie un sondage qui indique qu'une grande majorité d'Italiens sont opposés aux coupures publicitaires pendant les films. Parallèlement, un grand nombre d'intellectuels et de gens de cinéma, Fellini en tête, signent un appel en faveur du projet : Non si spezza una storia non si interrompe un'emozione. Bien que Walter Veltroni s'en défende, c'est bien d'un projet de loi anti-Berlusconi qu'il s'agit, puisque depuis 1984 Fininvest possède trois chaînes privées nationales qui ont un énorme besoin de programmes et qui puisent abondamment dans les réserves des sociétés cinématographiques italiennes. Il ne faut pas croire en effet que dans le monde du cinéma italien tous ont

2. Federico Fellini, Fare un film, op. cit., p. I38-139. 
les mêmes intérêts. Ainsi, si les auteurs voient leurs œuvres avilies par le traitement que la télévision commerciale leur réserve, les producteurs et autres ayant droit se félicitent de cette nouvelle source de revenus. Il faut dire que les intérêts en jeu son énormes, si l'on considère qu'en I988 les chaînes nationales ont diffusé plus de 6000 films et près de 550000 spots publicitaires, 7I \% desquels par les chaînes Fininvest ${ }^{3}$. Très pragmatiquement l'Anica (Associazione nazionale industrie cinematografiche e affini) et la Federazione Radiotrasmittenti négocient et aboutissent à un accord le 7 février 1989, qui prévoit sept interruptions de trois minutes maximum pendant la diffusion d'un film de ıoo minutes : une sur le générique initial, une autre sur le générique de fin, une troisième pendant l'intervalle et deux par partie du film. La durée totale de l'émission ne doit pas dépasser I2I minutes et l'histoire elle-même est interrompue quatre fois. La logique de la télévision commerciale l'a emporté. À ce propos, Giovanni Spagnoletti écrit :

[...] le passage historique de témoin du cinéma à la télévision, bien qu'en retard par rapport aux autres pays industrialisés, a d'autant plus agi en profondeur, avec une vitesse foudroyante et des effets destructeurs qui ont bouleversé le paysage cinématographique italien. Paradoxalement, le cinéma a été le cheval de Troie de cette opération, en contribuant à la prolifération du média rival, d'abord grâce à une consommation excessive de films programmés par les télévisions «libres»; ensuite par l'instauration d'un duopole mortel Rai-Mediaset (Berlusconi) qui, exception faite pour la jeune «mini Major» Cecchi Gori, a monopolisé - ou presque - une production cinématographique largement morcelée et artisanale ${ }^{4}$.

Mais l'argent des télévisions est indispensable aux producteurs italiens du fait de la faiblesse des recettes des salles (en 1990, il n'y a plus que 90 millions de spectateurs).

Tout ceci a de fortes conséquences sur les contenus et les formes des films. Fellini l'avait déjà remarqué : le danger du zapping contraint les auteurs à ne jamais faire faiblir le rythme du récit, les coupures fréquentes et la distraction possible du téléspectateur obligent à prévoir des moments de résumé de l'action. Lina Wertmüller, à cette époque, propose même de travailler sur des structures du récit qui prévoient les coupures publicitaires, à la façon qu'ont à l'époque les Américains de rythmer des séries telles que Columbo, Starsky et Hutch, etc.

3. Chiffres publiés par Videomagazine, janvier 1989.

4. Giovanni Spagnoletti, "Îles, I976-I998, ou Que s’est-il passé dans le cinéma italien?», dans Un'altra Italia. Pour une histoire du cinéma italien, Paris, Cinémathèque française, 1998. 
C'est incontestablement Maurizio Nichetti qui analyse le plus finement ce phénomène dans Ladri di saponette (1987), sans doute son chefd'œuvre. Le film enchâsse trois lieux idéaux, un film en noir et blanc, remake du chef-d'œuvre néoréaliste, le studio de télévision où l'auteur s'est rendu pour présenter le film en question et surveiller les coupures, le salon d'une famille de la petite bourgeoisie milanaise qui regarde le film à la télévision. Par un certain nombre de contaminations entre les publicités et le film, le récit du film en noir et blanc est totalement détourné et finit en mièvre comédie sentimentale, selon les vœux de la ménagère de moins de cinquante ans qui pourra ainsi aller se coucher sereinement. La fable est fort belle et montre comment les contenus et les formes des films sont désormais déterminés par les désirs de cette championne de la consommation dont il faut à tout prix éviter qu'elle change de chaine. Les auteurs se trouvent donc prisonniers de cette logique nouvelle. Mais Ladri di saponette a aussi un mérite supplémentaire : il montre un auteur, celui du film premier, aux prises avec une logique à laquelle il arrive intelligemment à s'adapter pour faire un film, parvenant même à le faire financer par ReteItalia, la société de production de Fininvest. Tout en démontrant, évidemment, combien ces financements entraînent des changements dans le métier - l'art - des cinéastes.

Á l'époque, l'argent des télévisions dans la production cinématographique n'est pas chose nouvelle. La Rai a produit ou coproduit de nombreux films dès les années 1970. Elle suivait en cela la tradition du sceneggiato des origines, l'adaptation d'œuvres littéraires classiques. Au début de la période qui nous intéresse, on retiendra Padre padrone des frères Taviani (1977), ainsi que L'albero degli zoccoli d'Ermanno Olmi (1978), tous deux primés à Cannes. Toutefois, la Rai rentabilise ses investissements dans le domaine du cinéma en faisant réaliser par des maîtres tels que Rosi ou Comencini des séries en plusieurs épisodes dont sont tirés aussi des montages plus brefs préalablement distribués dans les salles de cinéma ou destinés à l'exportation : on a ainsi Pinocchio (Luigi Comencini, 197I) Cristo si è fermato a Eboli (Francesco Rosi, 1979), Cuore (Luigi Comencini, 1987), La Storia (Luigii Comencini, 1987), et beaucoup d'autres. Par ailleurs, Rai et Fininvest investissent aussi dans des productions purement cinématographiques en payant à l'avance les droits d'antenne, par exemple. Ainsi, Reteitalia, entre 1983 et 1998 , intervient dans le financement de I53 films, parmi lesquels Bianca (Nanni Moretti, 1984), Gli occhiali d'oro (Giuliano Montaldo, 1987), La visione del Sabba (Marco Bellocchio, 1988), Dimenticare Palermo (Francesco Rosi, 1990), plus un certain nombre de premiers films. Cependant, la dominante de ce catalogue est le film medio, 
le film commercial, le plus souvent comique, avec des comédiens populaires. À partir de 1995, c'est la société Medusa qui se substitue à Reteitalia dans la production et la distribution cinématographique. La Rai, de son côté, crée Raicinema en 2000, ainsi que or Distribution. Ainsi les deux pôles télévisuels sont à parité et incontournables sur le marché cinématographique italien.

Il va de soi que ce nouvel ordre économique n'a pas été sans effet sur le contenu des films. Dissipons d'abord l'équivoque politique : si les productions financées par les sociétés de Silvio Berlusconi soutiennent son projet politique, ce n'est qu'indirectement, par le choix quasi exclusif du divertissement et non par propagande directe. En cela, il n'y a pas de grosse différence idéologique entre Medusa et Raicinema. Les «auteurs» y trouvent aussi leur place, à condition d'être confirmés, donc de produire des bénéfices. En revanche, l'argent des télévisions a causé la forme d'atonie que maints critiques ont relevée dans le cinéma des années 1980 et 1990 et qui substantiellement se poursuit. Dans le souci de financer des produits diffusables aux heures de grande écoute, les télévisions ont imposé au cinéma des codes consensuels de bonne conduite qui le dévitalisent. Mais, plus profondément, il y a surtout l'imposition d'une narration simplifiée à l'extrême. La télévision commerciale (la Rai vit aussi de la publicité) propose un flux d'images composées en histoires - spots publicitaires, fictions, informations, jeux - qui doivent être compréhensibles par le plus grand nombre et sans effort. La lisibilité est facilitée si on recourt à des schémas traditionnels, qui non seulement ne sont pas remis en cause, mais dont la crédibilité sera renforcée par cette nouvelle application. Pour simplifier au maximum la réalité, il convient qu'une histoire soit l'histoire de quelqu'un, qu'elle émane d'un seul point de vue - quelle n'ait qu'une vérité - et qu'elle ne réponde qu'à un seul principe causal. Il en va ainsi pour les publicités et pour les séries fondées sur quelques oppositions simples telles que masculin/féminin, bon/méchant, ami/ennemi et winner/loser. Lorsque le flux d'images devient trop complexe et requiert une plus forte attention - ou plus de culture - de la part du spectateur, le recours à la voix off devient systématique, ce qui renforce d'autant l'unicité du point de vue.

En ce sens, un film tel que Notte prima degli esami (Fausto Brizzi, 2006), coproduit par Raicinema, distribué par or Distribution est exemplaire. Sur la forme, d'abord :

- tourné en cinémascope, il peut parfaitement s'adapter à un format moins large car les bords de l'image ne sont pratiquement jamais utilisés ; 
- les épisodes sont montés chronologiquement et parfaitement délimités;

- la voix off du protagoniste intervient régulièrement au début pour fixer clairement les personnages et les rapports qu'ils entretiennent avec lui;

- l'épisode d'ouverture est particulièrement drôle et inattendu, le générique n'intervient qu'après;

- les personnages sont tous beaux et sympathiques, ceux qui ne le sont pas vont le devenir;

- il ne manque pas d'épisodes dramatiques, mais ils sont aussitôt résorbés.

L'histoire, quant à elle, est centrée sur le personnage de Luca qui a injurié dans le premier épisode son professeur de lettres sans savoir qu'il allait être membre du jury du bac; d'où un programme narratif fort simple : Luca réussira-t-il à remonter ce handicap? Par ailleurs, pour Luca la période des révisions se double d'une quête, celle de Claudia, la fille connue le premier soir puis perdue et qui n'est autre que la fille de son professeur de lettres.

Le film raconte un rite de passage avec drames et bonheurs de la vie quotidienne sur un fond de joie de vivre et de ciel toujours serein, qui met tout le monde de bonne humeur. Enfin, cette histoire située à la fin des années I980 cible un public d'adolescents ainsi que leurs parents qui avaient l'âge des personnages à l'époque représentée.

Tout cela entraîne une grande perméabilité entre le monde du cinéma et celui de la télévision. Les cinéastes, les scénaristes et les comédiens passent aisément d'un mode de production et de tournage à l'autre : derniers exemples, Alessio Boni et Violante Placido interprètes de Guerre et paix, Roberto Faenza, réalisateur de I viceré, en double version cinéma et minisérie télévisée. On annonce aussi la prochaine sortie de Sangue pazzo, de Marco Tullio Giordana, une sorte de remake de Telefoni bianchi (Dino Risi, 1976) avec Monica Bellucci, Luca Zingaretti et Alessio Boni. Il s'agit d'une co-production entre une société italienne, Bibì Film, une société française, Paradis Film, Raicinema et Rai Fiction. Le film sortira d'abord en salle puis en mini-série à la télévision.

Grâce à ce système de production qui se met en place, on assiste à un renouveau des genres, noir et comédie à l'italienne essentiellement. Ce sont souvent des films de qualité, attractifs et spectaculaires, qui ont permis au cinéma italien de récupérer des parts de marché importantes 
sur son territoire. Des films tels que Romanzo criminale (Michele Placido, 2005), Arrivederci amore ciao (Michele Soavi, 2006) ou ancore Notturno bus (Davide Marengo (2007) côté polar, Manuale d'amore (Giovanni Veronese, 2005) ou Il mio miglior nemico (Carlo Verdone, 2006) côté comédie, ont manifesté un véritable renouveau du film de divertissement de qualité.

Mais il reste peu de voies pour la recherche personnelle hors de ces cadres stricts. Bien qu'il existe une contribution que l'État verse à des films dits d'interesse culturale sur lecture et évaluation du scénario, bien que les nouvelles caméras digitales permettent de réaliser des films avec de faibles budgets, le problème essentiel reste celui de l'exposition de ces films «différents» au grand public. Chaque année apporte son lot d'œuvres intenses, intéressantes, délicates, fragiles donc et marginales car les écrans sont monopolisés par les blockbusters nationaux ou internationaux. Beaucoup de ces films ne sont visibles que dans les festivals ou dans quelques salles d'essai des grandes villes. Qui connaît Onde (Francesco Fei, 2004), Sotto il sole nero (Enrico Verra, 2004), Mater natura (Massimo Andrei, 2005), Come l'ombra (Marina Spada, 2006), Billo, le grand Dakhaar (Laura Muscardin, 2006), Jimmy della collina (Enrico Pau, 2006), Le ferie di Licu (Vittorio Moroni, 2007) ? Et la liste pourrait être bien plus lingue...

J'emprunterai le mot de la fin à l'un des films les plus beaux que le cinéma italien ait donné ces dernières années. Il s'agit de Valzer de Salvatore Maira, présenté à Venise en 2007. L'un des personnages dit à la protagoniste - véritable alter ego de l'auteur : «la gente come te è destinata a rimaner sola». Solitude, désolation, enfermement, semblent bien la destinée commune de ces films d'auteur qui essayent de raconter le monde sans se référer aux narrations que la société du spectacle solidement installée impose de façon toujours plus tyrannique. 
